\title{
DÜBLIN
}

Technological University Dublin

ARROW@TU Dublin

\section{In situ Image Processing and Data Binning Strategy for Particle Engineering Applications}

\author{
Toufic El Arnaout \\ Technological University Dublin, toufic.elarnaout@tudublin.ie \\ Patrick J. Cullen \\ University of Sydney, Australia
}

Follow this and additional works at: https://arrow.tudublin.ie/schfsehart

Part of the Biochemical and Biomolecular Engineering Commons

\section{Recommended Citation}

El Arnaout, T. and Cullen, P.J. (2020), In situ Image Processing and Data Binning Strategy for Particle Engineering Applications. Chem. Eng. Technol., 43: 1618-1629. https://doi.org/10.1002/ceat.201900311

This Article is brought to you for free and open access by the School of Food Science and Environmental Health at ARROW@TU Dublin. It has been accepted for inclusion in Articles by an authorized administrator of ARROW@TU Dublin. For more information, please contact arrow.admin@tudublin.ie, aisling.coyne@tudublin.ie, gerard.connolly@tudublin.ie.

Funder: EC's Seventh Framework; Science Foundation Ireland

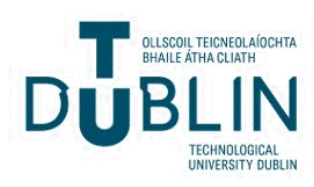


Toufic El Arnaout ${ }^{1,2, *}$

Patrick J. Cullen ${ }^{3}$

Supporting Information

available online

\section{In situ Image Processing and Data Binning Strategy for Particle Engineering Applications}

Crystallization control can be improved through real-time monitoring technologies. Here, a workflow is demonstrated on rapid batch cooling crystallization of $L$-glutamic acid. First, in situ images were generated using video microscopy sensors and analyzed, by employing a single, rapid macro code to extract particle data descriptors. A binning procedure (over time) was performed, where every data point represented the counts of particles within a specific size or shape range per 100 images. This binning method was found more informative in tracking of the populations compared to whole image averages or individual particle datapoints. This study provides a step-by-step guide towards improving mechanistic modeling, control via feedback, automation, and continuous manufacturing for Industry 4.0 .

Keywords: Crystal size-shape, Data processing, Process analytical technology, Two-dimensional image analysis

Received: June 02, 2019; revised: February 20, 2020; accepted: April 21, 2020

DOI: 10.1002/ceat.201900311

\section{Introduction}

\subsection{Brief Review of Process Analytical Technology for Continuous Monitoring}

Designs and data-driven activities for the industries of the future have been rapidly emerging, underpinned with the concepts of continuous manufacturing and quality by design (QbD). Process analytical technology (PAT) permits the monitoring of most process steps and facilitation of feedback strategies $[1,2]$. The paradigm can enhance troubleshooting and process understanding, and reduce drifts and batch-batch variability, waste, cycles and time. Several domains may also benefit from monitoring in solution, such as systems of liquid and gas (with and without solids), liquid and emulsions or bubbles, and liquid and solids (particles, fibers, granules).

In situ sensors (immersible) may be based on different measurement methods available, such as the laser backscattering (for the chord length (CLD)) and video microscopy for size and shape. All techniques have challenges and limitations, with the former including challenges caused by particle transparency, chord splitting [3,4], chord concatenation [5], and data skewness when attempting to relate chord length to size [6]. The latter, i.e., microscopy, may be also restricted in efficacy due to system resolution, limited or negligible performance above certain concentrations $(>2-8 \%$ ), and, when it comes to data processing of the acquired images, there are also limitations related to particle overlap [7], difficulty with edge detection [8], watersheding, and segmentation [9].

\subsection{Towards Mechanistic Models Incorporating Size-Shape Factors}

Process control can be enhanced by continuously measuring variables for the species (e.g., particles, crystals), their media, and the effect of additives [10]. In some cases, temperature cycling for an enhanced control is also employed [11]. Population balance models (PBMs) and equations (PBEs) are often of $1 \mathrm{D}$, based only on length, with simplified assumptions about the particle populations, shape, and homogeneity. Recently, improved models related to crystal size were proposed [12]. Many reviewed models do not include shape information despite its importance and influence on material properties.

Certain equations do consider a general shape factor for all particles in the solution, or that particles are spherical in shape, where the length equals to the diameter. Some models include

\footnotetext{
${ }^{1}$ Dr. Toufic El Arnaout

Technological University Dublin - City Campus, School of Food Science and Environmental Health, Marlborough st, Dublin 1, Ireland.

${ }^{2}$ Dr. Toufic El Arnaout toufic.arnaout@kappacrystals.com Kappa Crystals Ltd, Dublin, Ireland.

${ }^{3}$ Prof. Patrick J. Cullen

University of Sydney, School of Chemical and Biomolecular Engineering, Sydney, Australia.
} 
basic 2D information (width and length), which can be further optimized if all shapes and dimensions are measured, as not all particles are cylindrical/rectangular in shape. In the literature, kinetic studies employ $k_{\alpha}$ or $k_{v}$ in the equations, representing the surface shape factor and the volumetric shape factor, respectively (Eqs. (1) and (2)).

$k_{\alpha}=($ Area of structure $) /(\text { Crystal length })^{2}$

$k_{\mathrm{v}}=($ Volume of structure $) /(\text { Crystal length })^{3}$

The values of $k_{v}$ and $k_{\alpha}$ depend on the crystal habit (e.g., shape), e.g., spherical $\left(k_{v}=\pi / 6\right.$ and $\left.k_{\alpha}=\pi\right)$, cubic $\left(k_{v}=1\right.$ and $\left.k_{\alpha}=6\right)$, average needle $\left(k_{v}=0.04\right)$, etc. Practically, an aspect ratio of $0.6-0.8$ was found as a typical median range for most particles of active pharmaceutical ingredients (APIs) [13]. A mass balance model for continuous batch crystallizers describing the concentration evolution of solute is according to Eq. (3) [14]:

$\frac{\mathrm{d} C}{\mathrm{~d} t}=-3 \rho k_{\mathrm{v}} h \int_{0}^{\infty} f L^{2} G \mathrm{~d} L$

In Eq. (3), $C$ is the solute concentration (masssolvent basis), $p$ is the crystal density, $G$ is the growth rate, $f$ is the crystal distribution function, $h$ is a conversion factor (slurry volume per solvent mass), and $L$ is the crystal size.

As for 2D PBMs, these have been increasingly employed to study prominent $2 \mathrm{D}$ shapes such as needles [15]. Volmer's equation was proposed to describe the primary nucleation rate and power law for the secondary nucleation rate. The following expression of crystal population volume fraction includes the shape factor:

$V_{\mathrm{c}}=k_{\mathrm{v}} \int_{0}^{\infty} \int_{0}^{\infty} L_{1} L_{2}^{2} n(L, t) \mathrm{d} L$

Additional equations for a $2 \mathrm{D}$ system [16] to describe the growth $(G)$ or dissolution $(D)$ with exponents, (e.g., dissolution exponents $d_{\mathrm{L}}$ and $d_{\mathrm{W}}$ ), are as follows [17]:

$G_{\mathrm{L}}=k_{\mathrm{g}_{\mathrm{L} 0}} \exp \left(-\frac{\Delta E}{R T}\right) S^{\mathrm{g}_{\mathrm{L}}}\left(1+\delta_{\mathrm{gL}} L\right)$

$G_{\mathrm{W}}=k_{\mathrm{g}_{\mathrm{W} 0}} \exp \left(-\frac{\Delta E}{R T}\right) S^{\mathrm{g}_{\mathrm{w}}}\left(1+\delta_{\mathrm{gW}} W\right)$

$D_{\mathrm{L}}=\frac{k_{\mathrm{dL}}(-S)^{\mathrm{d}_{\mathrm{L}}}}{L^{\gamma}}$

$D_{\mathrm{W}}=\frac{k_{\mathrm{dW}}(-S)^{\mathrm{d}_{\mathrm{W}}}}{W^{\gamma}}$

Here, $S$ is the absolute supersaturation. Size dependency is defined through $\delta_{i j}$ and $\gamma$. The growth rates $(G)$ along the width $(W)$ and length $(L)$ dimensions are indicated; $k$ is the rate constant. Other factors are the activation energy $(E)$, gas constant $(R)$, and temperature $(T)$.

\subsection{Video Microscopy Sensors for Size-Shape Characterization}

Images provide several advantages for particle characterization: (a) direct observation allows an evaluation of a multitude of factors; (b) not only the size but shape information [18]; (c) analysis of texture; (d) digital information for processing and binary analysis; (e) extraction of numerical data and particle analysis. 2D images for moving particles can be obtained directly from the process (Fig. 1) by employing engineered, immersible microscopy sensors using several optical methods $[19,20]$, while the full $3 \mathrm{D}$ morphology is possible via offline

a)

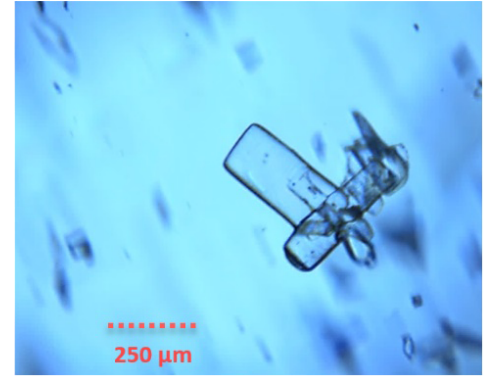

b)

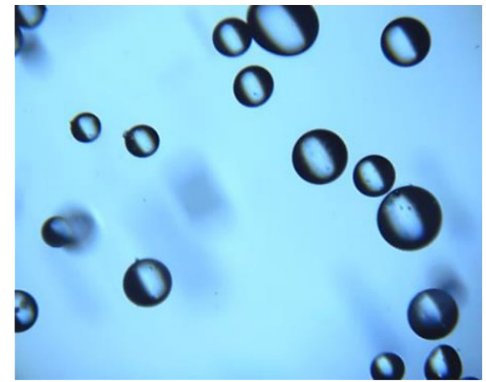

C)

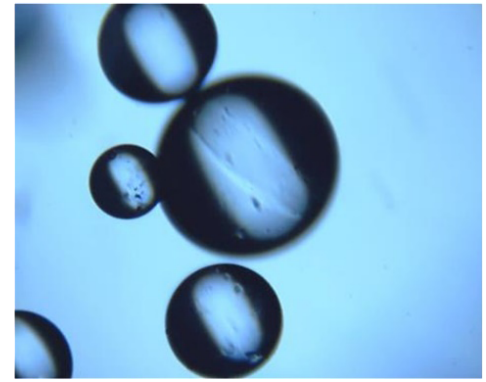

d)

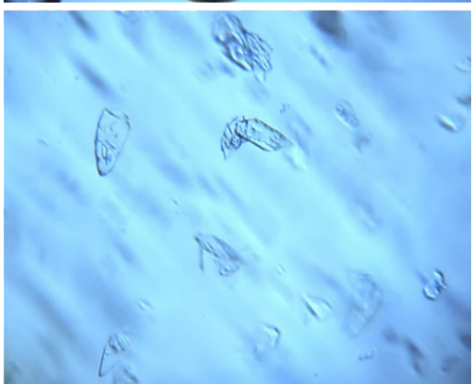

Figure 1. In situ images of particles captured directly in solution during stirring using an immersible imaging probe (video microscopy sensor). Left: bright field mode. Right: relief contrast optical mode. (a) Benzoic acid; (b) polydisperse particle standard $1(50-350 \mu \mathrm{m})$; (c) polydisperse particle standard $2(150-650 \mu \mathrm{m})$; (d) fosfomycin. 
controlled setups [21]. In one of the in situ sensors (Fig. 2), a relief contrast method [22] was incorporated, which allows under certain conditions an enhancement of the observation of textures and thin features, and using a more flexible assembly than traditional differential interference contrast (DIC).

\subsection{Approach for Background Correction and Data Binning}

Extracting particle information from the acquired images is not straightforward due to the variations in background intensities, shadows from particles out of focus, gradients of color, and brightness, blurriness, and noise. Additional challenges are due to particle overlap and difficult to interpret or missing signals. The first set of challenges are shown to be overcome in this paper, however, the second set are mostly case-specific, solved for basic, preknown shapes only.

Having tested several open-access functions for background correction or normalization for this study and in previous ones $[19,20]$, one of the most useful methods that was identified is called the 'rolling ball' method [23]. The approach can autoadjust to gradients within the same image, random depths, and large variations in the $2 \mathrm{D}$ baseline. The technique also permits smoothing in parallel, does not require a time-zero blank image normalization, which is generally not compatible with in situ images, and can be applied directly and independently from one image to another. It was developed as a code by M. Castle and J. Keller, University of Michigan, and is available in Image J [24].

An important improvement known as the 'sliding paraboloid' (by M. Schmid) has also been implemented, which is optimized for many slopes and color variations, and with better lateral extensions for varying intensities and shapes. In certain versions of Image/Fiji, functions also permit the selection of the radius of the moving baseline-correction-ball/parabolae, background properties, with considerations for color channels. An example is presented for the background correction of an in situ image containing a strong slope due to the relief contrast prism used, with color and brightness variations (Fig. 3). A digital contrast enhancement is also important.

\subsection{The Importance of this Study}

The application of in situ microscopy sensors is demonstrated on both static drops and batch cooling crystallization reactors. Furthermore, solving challenging datasets with difficult image a)

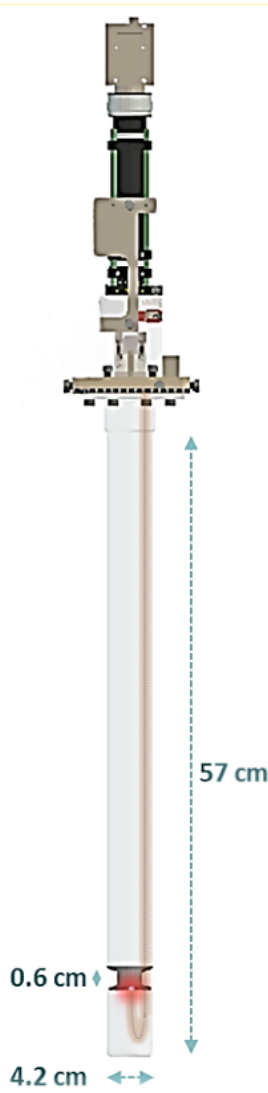

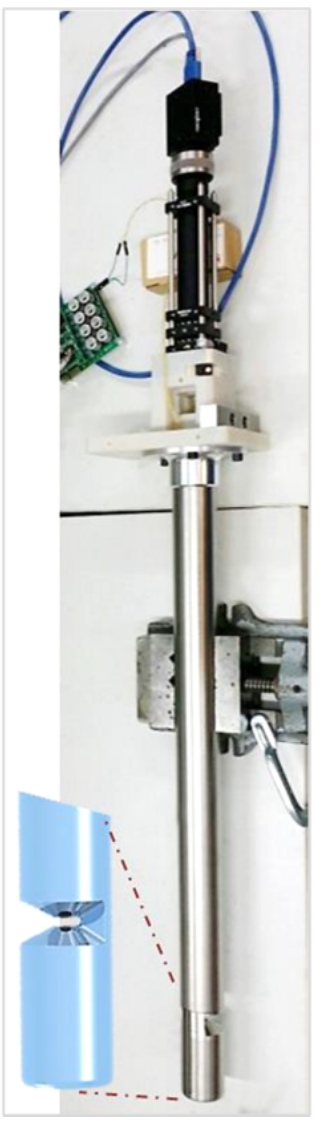

b)

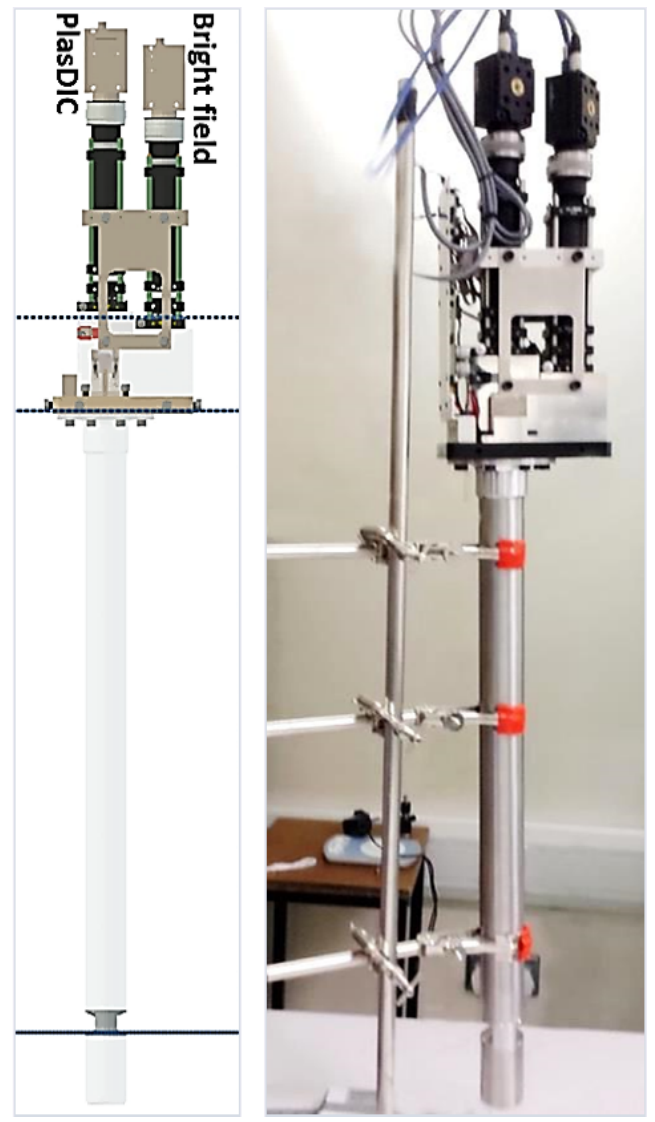

Figure 2. Video microscopy probes for monitoring particles in solution (in-line, in situ, real-time PAT). (a) A simple design with backlight illumination via an optical fiber, permitting visualization in bright field mode. (b) An upgraded design incorporating a relief contrast optical method for the enhanced visualization of texture and thin/transparent particles. Some figures were adapted $[19,20]$. 


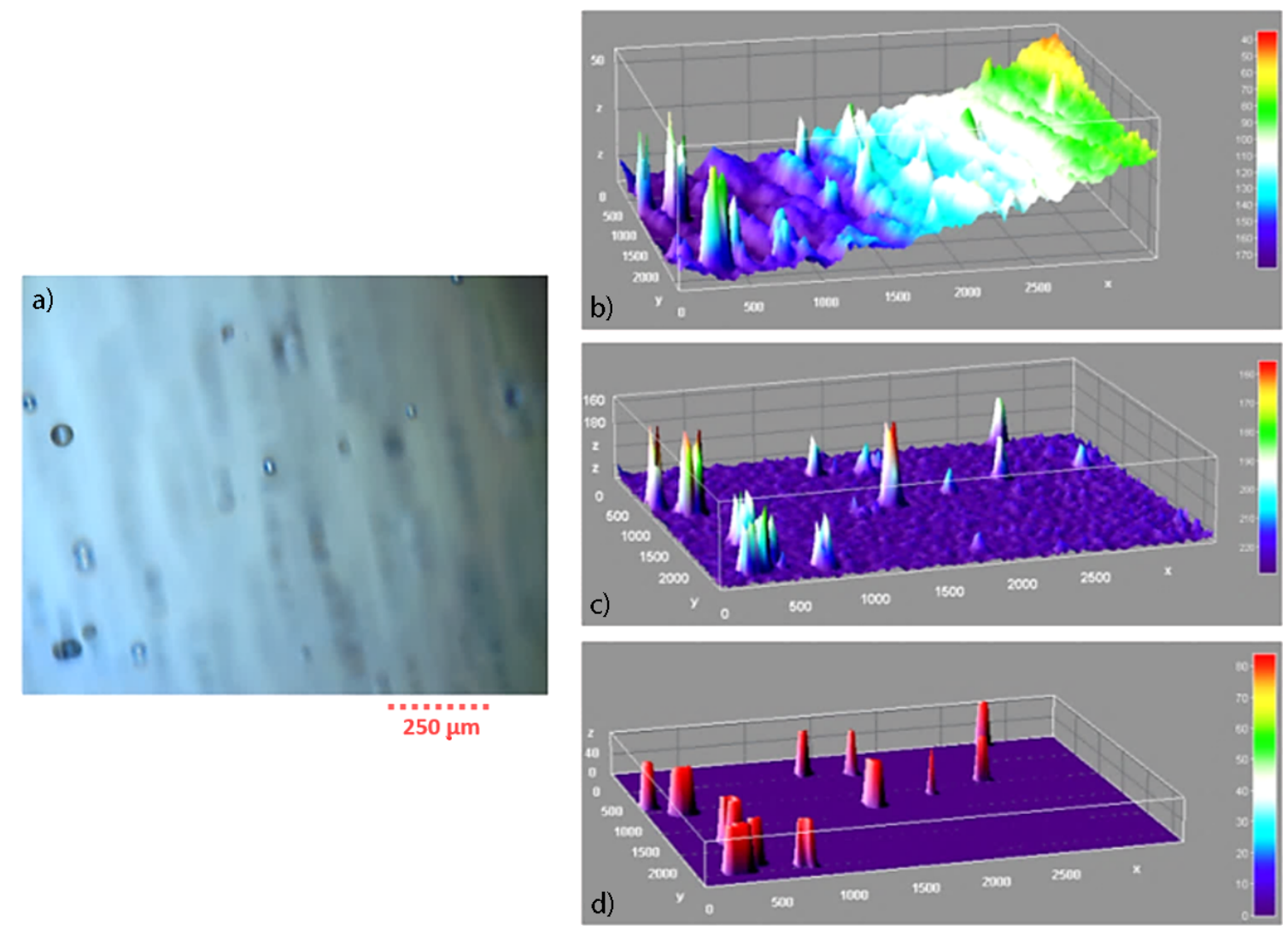

Figure 3. Processing images from a relief contrast setting containing gradients of color, intensity, and brightness. (a) Raw image of particle standards $(15-150 \mu \mathrm{m}) ;(b-d) 3 D$ surface plots. (a) Original image showing the gradient; (b) image following background correction and normalization operations; (c) final binary image after noise correction.

backgrounds and strong contrast gradients is proven step by step with more details than in our previous studies to extract particle size-shape information. The development of a single macro-code applicable on an entire bright-field generated image dataset without manual intervention is demonstrated and the code included in this paper, and similarly for the reliefcontrast image dataset.

Furthermore, following the generation of binary data from image analysis, challenges of data management and binning were addressed, with comparisons against other representation methods. For instance, in a 2-h experiment with images recorded at a rate of 1 per $5 \mathrm{~s}$, the total number of images will be 1440 images. If each image contains on average 30 particles (including microparticles of $5-15 \mu \mathrm{m}$ ), then the total number of particles will be 43200 . Therefore, to identify an ideal strategy for tracking sizes, shapes, and their distributions, several data presentation approaches were compared:

(1) Plotting a single average of size or shape per image (one data point per image); (2) plotting the size or shape of every particle (one data point per particle) in every image; and (3) plotting the frequencies of particles belonging to specified data bins ( $x-y$ intervals or ranges) of size or shape, in a $Z$ number of images at a time (one data point for the counts of particles within a specific size/shape range, in a $Z$ number images) (Fig. 8).
Potential applications and the usefulness of the generated data are also discussed in terms of automation, industries of the future, continuous manufacturing, and mechanistic control based on the quality of measurements and real-time analysis of shape, in situ.

\section{Materials and Methods}

\subsection{Chemicals and Particles}

For generating the images in Fig. 1, the following steps were employed: (1) dried crystals of fosfomycin in powder form were stirred at $0.5 \% \mathrm{w} / \mathrm{v}$ in isopropanol; (2) benzoic acid crystal powder was stirred at $1.5 \% \mathrm{w} / \mathrm{v}$ in water; (3) polydisperse particle standard was stirred at $1 \% \mathrm{w} / \mathrm{v}$ in water using $50-350 \mu \mathrm{m}$ or $150-650 \mu \mathrm{m}$ spheres (Whitehouse Scientific, LGC Standards UK, Cat. WS-PS227 and WS-PS237). For Fig. 3, $15-150 \mu \mathrm{m}$ spherical particles at $1 \% \mathrm{w} / \mathrm{v}$ were also stirred in water (Cat. QAS3002, Malvern). In addition, the following chemicals (Sigma-Aldrich) were used: taurine (Cat. W381306-5KG-K), paracetamol (Cat. A5000-1KG), $L$-glutamic acid (Cat. W328502-5KG-K), and D-mannitol (Cat. M9647-1KG). 


\subsection{Crystallization of Glutamic Acid}

Crystallization in water was carried out from supersaturation at high temperatures of $60-65^{\circ} \mathrm{C}$ over several hours [19]. Initially, $24 \mathrm{~g}$ was added into $700 \mathrm{~mL}$ water. Crystals began forming at $\sim 48^{\circ} \mathrm{C}$ (Fig. $8 \mathrm{~d}$ ). Recording while temperature was dropping continued for $\sim 2 \mathrm{~h}$, and during that time, once it reached $42.7^{\circ} \mathrm{C}$, it was then increased. This protocol results initially in a fast crystallization into single small needles $(5-30 \mu \mathrm{m})$ with the multi-needle aggregate shapes observed in Fig. 8a becoming dominant. Image recording was performed over $\sim 2 \mathrm{~h}$ when the crystallization started, at a rate of one image per $5 \mathrm{~s}$.

\subsection{Probe Sensors}

The probe sensors (Fig. 2) using both the bright field and relief contrast methods were developed by VTT (Finland), Innopharma Technology (Ireland), TU Dublin [19,20], and partners of the CrystalVis EU FP7 project.

\subsection{Algorithms and Data Processing}

The macros were built based on available functions/scripts in ImageJ/Fiji. Overall, the simplest and most efficient algorithm developed for processing bright field images in situ (Figs. 7 and 8 ) is the following (from raw image to particle analysis tables):

$\operatorname{run}\left(" 32-b i t^{\prime \prime}\right)$;

run("Enhance Contrast...", "saturated $=0.4$ ");

run("Subtract Background...", "rolling=200 light sliding");

run("Make Binary");

run("Remove Outliers...", "radius=20 threshold $=10$ which=

Dark");

run("Dilate");

run("Fill Holes");

run("Make Binary");

run("Set Scale...", "distance=1 known=0.377 unit=unit");

run("Set Measurements...", "area standard min center perimeter bounding shape feret's integrated median area_fraction limit display redirect $=$ None decimal $=2 ")$;

run("Analyze Particles...", "display exclude summarize record");

The other macro, for processing the images containing the gradient and relief contrast (Figs. 3, 6, and 7) is the following:

run("Enhance Contrast...", "saturated=0.4 equalize");

run("Subtract Background...", "rolling=200 light separate sliding");

run("Make Binary");

run("Remove Outliers...", "radius=20 threshold=10 which=

Dark");

run("Dilate");

run("Fill Holes");

run("Make Binary");

run("Set Scale...", "distance=1 known=0.377 unit=unit");

run("Set Measurements...", "area standard min center perimeter bounding shape feret's integrated median area_fraction limit display redirect $=$ None decimal $=2 ")$;

run("Analyze Particles...", "display exclude summarize record");
The average time to process an image was $10-30 \mathrm{~s}$. The first macro was more rapid as it only requires a 32-bit greyscale image rather than colored, and for the background correction step there is no color separation. The background correction is generally the most time-consuming step.

Following the generation of the tables of (i) summary and averages per image and (ii) details per particle per image, the data was exported to Microsoft Excel. Calculations of frequencies and distributions, and plotting of results was then performed. For the graph in Fig. 8b, the average sizes (Feret) and counts per image were plotted with each data point representing an image. For the graph in Fig. 8c, the sizes (Feret) of particle by particle (all particles in all images) were plotted. For the graph in Fig. 8d, the distributions (frequencies) based on the counts in specific size intervals in every 100 images were plotted. Therefore, in Fig. $8 \mathrm{~d}$, each data point represents the frequencies for a particular size range in every 100 images, and each curve is related to the evolution of that specific size range in every 100 images.

To generate the graphs in Figs. 4 and 5, in addition to the counts per image, the overall averages per image (image by image) were plotted for the size (Feret's diameter), shape circularity, and shape solidity. Here, circularity is $4 \pi \frac{[\text { area }]}{[\text { perimeter }]^{2}}$. Note that for paracetamol (acetaminophen) the strong relief contrast images containing a strong gradient were processed based on similar protocols of signal combination [20]. Basically, each image is first duplicated, then one copy is processed based on the dark background correction for the bright pixel extraction and threshold, while the second copy is processed based on the light background correction for the dark pixel extraction and threshold. Both binary images are then combined into one image and analyzed.

\section{Results and Discussion}

\subsection{Application on Drop-Sized Trials}

Drops on a slide were imaged, with crystal growth of either taurine with bright field (Fig. 4) or paracetamol (i.e., 4-acetamidophenol or acetaminophen) with relief contrast (Fig. 5). It was possible to enhance the contrast of the images and to correct the backgrounds in both experiments. For instance, weak inhomogeneities of brightness and intensity surrounding the taurine crystal signals were corrected, including faint contours of the drop boundary. For the paracetamol crystals, corrections were possible for the strong contrast and color gradients, with both the bright and dark signals successfully extracted and merged throughout the gradient.

Particle segmentation presents a separate challenge and was not dealt with here due to the random sizes, shapes [25], and overlaps. However, in this case, particle tracking and counting was adequate up to a certain concentration. Taurine crystals decreased in shape circularity and solidity (Fig. 4), while such shape changes were less evident for the paracetamol crystals (Fig. 5) which had only a small increase in size during the experiment. As most particles had a similar shape, the trend line representing the size may be used to directly estimate the trend 

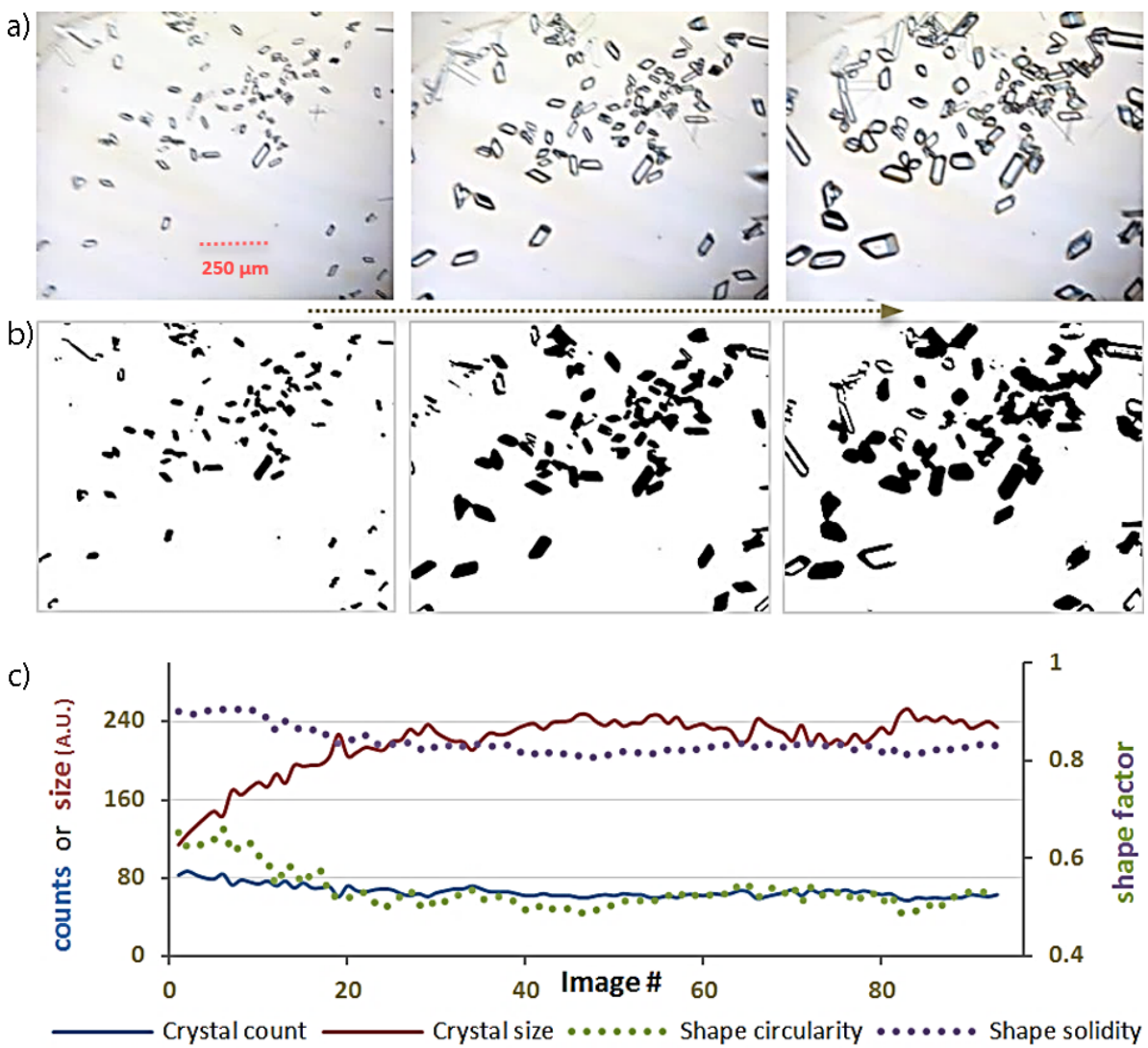

Figure 4. Image processing and size-shape monitoring (in bright field) of taurine crystals in a drop. (a) Raw images; (b) binary images after processing; (c) tracking of counts and averages per image of the size (Feret's diameter) and shape (circularity and solidity factors). Each size A.U. is equal to $0.377 \mu \mathrm{m}$ (e.g., 100 A.U. $=37.7 \mu \mathrm{m}$ ).

line for volume, and here it indicates how, although the particle counts remain constant, the size and/or the volume tracking can be independent.

An additional challenge is the extraction limitation of the algorithms in terms of 'particle completeness'. Particularly with the larger and more transparent particles, certain edges are often observed at a contrast similar to that of the background. The resulting binary information for such particles is an opened shaped such as a U-shape (Fig. 4).

Figs. 4 and 5 present an image-by-image statistical analysis of the overall size or shape averages for all particles (one data point per image). However, for longer studies (Sect. 3.3), additional interpretation and binning are necessary as otherwise the overall averages will be likely similar and strongly weighted by microparticles or randomly extracted signals.

\subsection{Application on Batch-Cooling Reactors}

The ultimate aim of the present experiment is the implementation of such techniques into large-scale reactors, in which the probes (Fig. 2) are fully immersed without the operator's physical access or control over the sample. For the previous section, the samples were in one (focal) plane placed on a $2 \mathrm{D}$ slide, here, the crystals are free in a $3 \mathrm{D}$ volume fully occupying the sampling gap of $6 \mathrm{~mm}$ at the distal part of the probe, to demonstrate the real application of the strategy in a reactor, where the acquired images are expected to contain more noise and stronger blurriness, with particles in/out or partially in focus and rapidly moving (Fig. 7).

Furthermore, while there are advantages for texture and contrast analysis, the application of the relief contrast method poses another challenge for image processing. The images contain dark sides, in addition to rich and altered color information (Fig.6), possibly due to the changed properties of the light, phases, and refractive indices throughout the sampling gap, the solvent and multiple crystals at varying planes, and influenced by the relief contrast prism properties. Fortunately, and as described in Sect. 1.4, the macros employed permitted the correction of the hue and saturation. In such macros, the function of digital enhancement of contrast with the option of 'histogram equalization' [26] was found to be particularly useful for adjusting pixel intensities and enhancing details (Fig. 6d). Of note is that for our case, the 'Equalization' function was not necessary for the bright field images, for which the general process was found to be faster, as starting with a conversion to a simpler 32-bit greyscale version in ImageJ was sufficient.

For the relief contrast images, a specific order and choices of executed functions was necessary (Fig. 6). Starting with the colored raw images (Fig. 6a), the first attempt (Fig. 6b) beginning with 32-bit greyscale conversions, as an attempt to speed up the process for large datasets, resulted in the loss of important color information associated with the contrast and gradient, but also transformed large areas of the images, particularly dark components, into 1000s (and at times 100000 s) of pixels or groups of pixels (in the final binary image) that massively inflated the output table of statistics. In the second example (Fig. 6c), this issue was overcome with the contrast enhancement function, even though the 32-bit conversion was still used. However, a large portion of the information necessary to extract, in particular from the dark side of images, was missing. This was solved in the third example, by starting with the colored image versions and carrying out the contrast enhancement (Fig. 6d), which demonstrated an improved signal extraction and binary recovery. The sliding paraboloid background correction method was used with the parallel function of color/ hue correction also activated. Further details about the challenges to initially solve this type of datasets are given in Fig. S1 in the Supporting Information, with side-by-side comparisons 

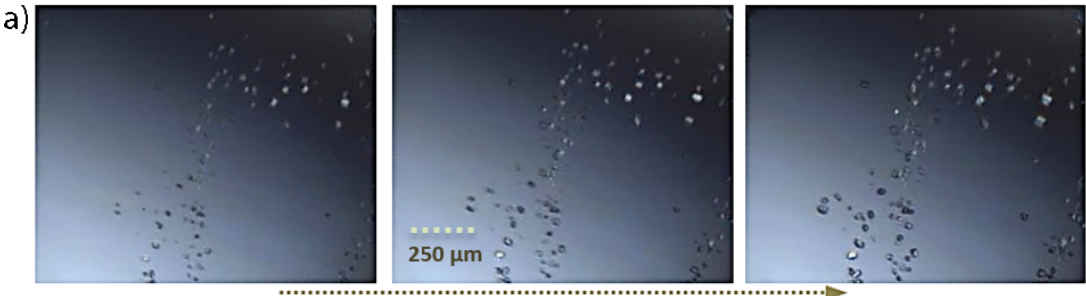

b)

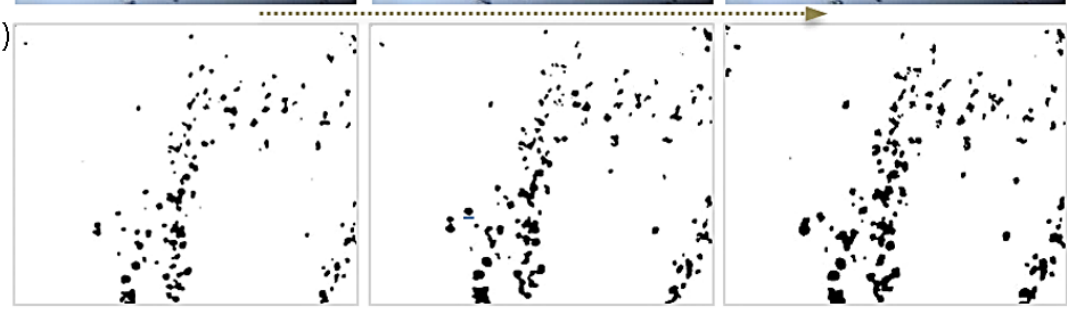

c)

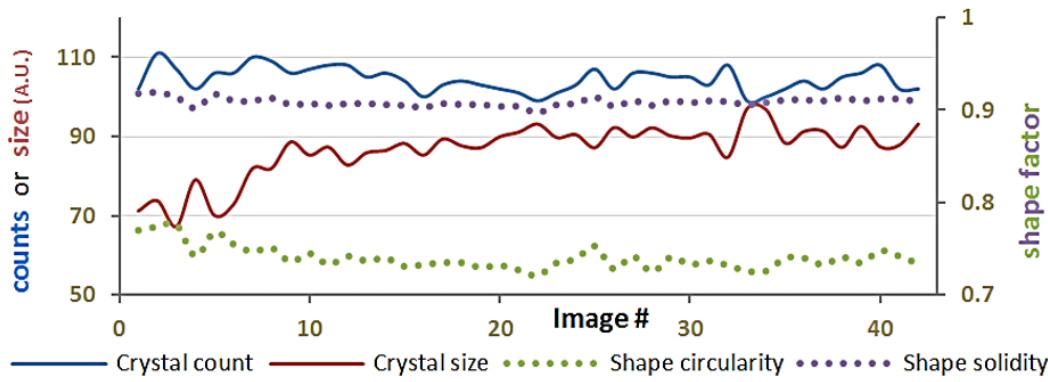

Figure 5. Image processing and size-shape monitoring (at strong relief contrast) of paracetamol crystals in a drop. (a) Raw images; (b) binary images after processing; (c) tracking of counts and averages per image of the size (Feret's diameter) and shape (circularity and solidity factors). Each size A.U. is equal to $0.377 \mu \mathrm{m}$ (e.g., 100 A.U. $=37.7 \mu \mathrm{m}$ ). between different scenarios and order of function execution, including that of the subtraction of time-zero blank images.

The challenge of optimal binary conversion of images for particle analysis was reasonably accomplished for both bright field and relief contrast image datasets (Fig.7). The two respective macros of sequences of functions used are indicated in Sect.2. In particular, processing of bright field images was more rapid due to the bypassing the need for correction of the colored version of images through the use of 32-bit formats. The advantage of both macros is the feasibility to automatically correct all images within a dataset, whatever the size (including microparticles [27]) or shape distribution, without causing noise or unusable regions in the binary images. However, binary particle completeness was an issue in some cases, but there was generally acceptable recovery of the overall information. This is particularly true when compared to other methods such as the chord length determination via lasers with the scan occurring through only part of the particle [28] (Fig. 9). While in this case a rich set of parameters is accessible including, at least, the binary extraction and measurements of different parts along many chords. However, an issue is the visual a)

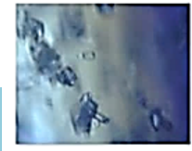

b)

c)

d)
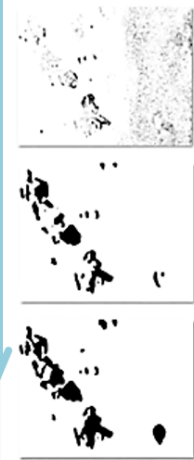
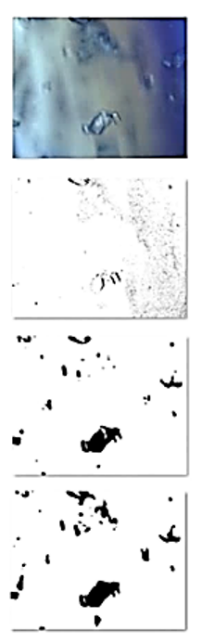
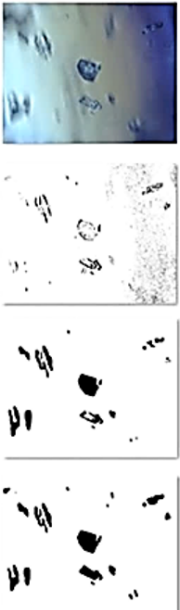
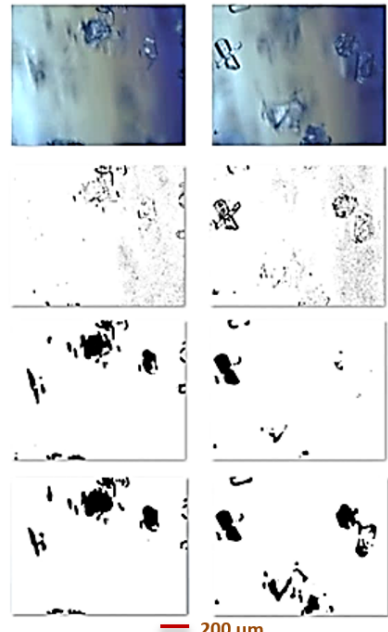

1.

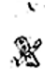

成

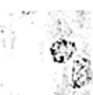

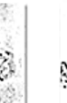

\section{8}
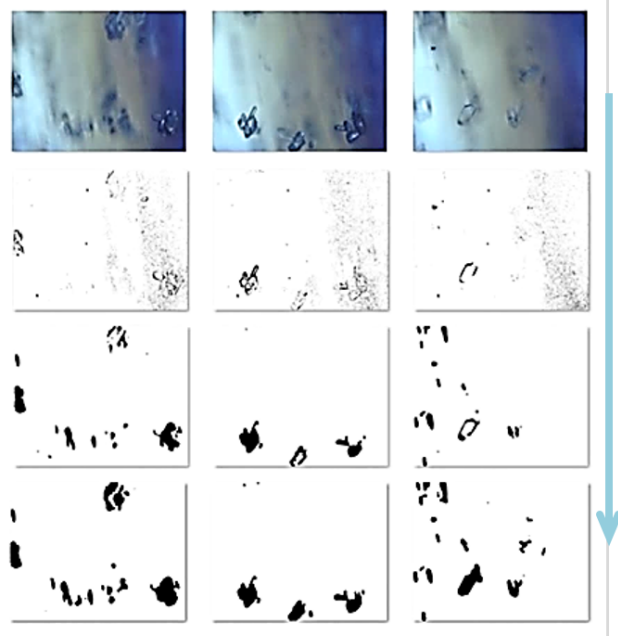

Figure 6. Processing an entire image dataset from a crystallization reactor using a single code.

(a) Raw images recorded in situ of fast cooling adipic acid batch crystallization (in water) using relief contrast optical setting. (b) First attempt based on an algorithm that only extracts parts of the particle signals and is not suitable for the dark parts of the image/gradient, resulting in $1000 \mathrm{~s}$ of noise items. Images were first converted to 32-bit greyscale to reduce computing time. (c) Second attempt based on an algorithm able to solve the noise exhibited in (b), but that only extracts the signals of certain (or part of) particles. Images were also converted first to 32-bit greyscale, but followed by contrast enhancement, before background correction. (d) A satisfactory approach for object extraction. Colored image formats were used without conversion to greyscale, followed by contrast enhancement with histogram equalization and a sliding paraboloid background correction with the color separation function activated. 
a)

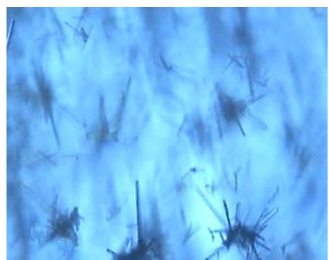

b)

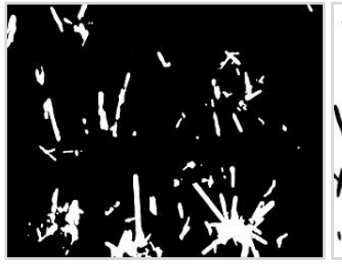

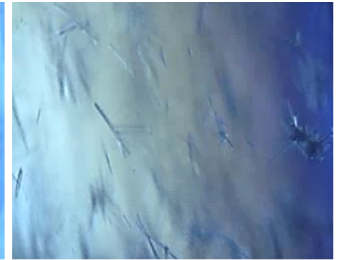
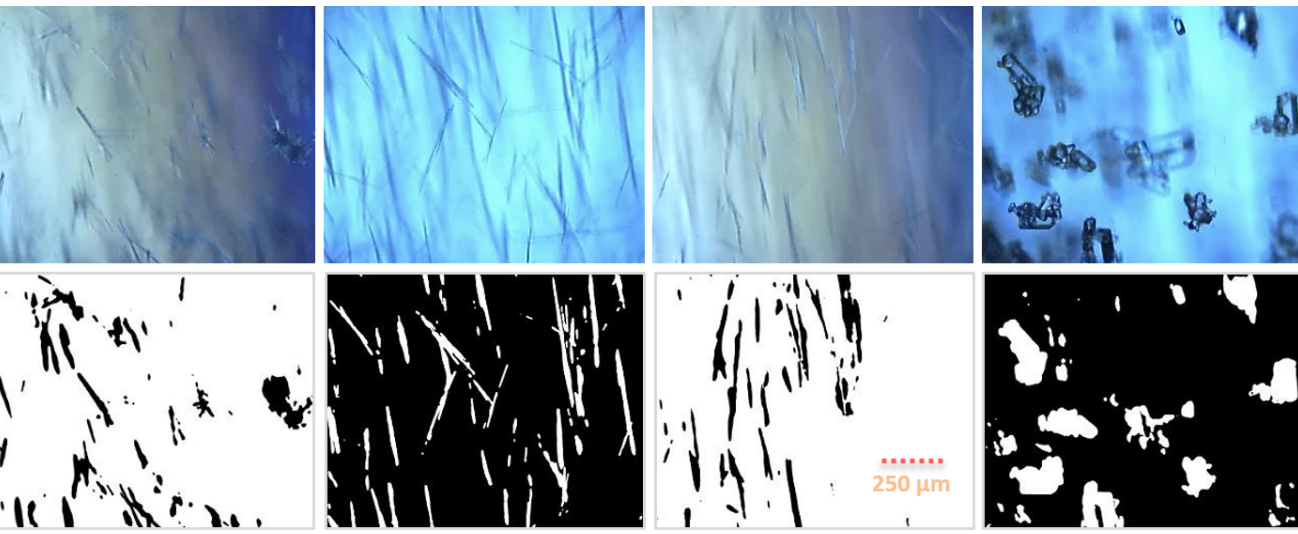

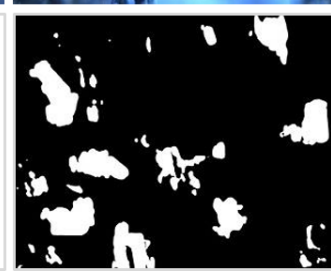

Figure 7. Processed video microscopy images of different crystal sizes and shapes. (a) Raw in situ images (1, 3, and 5: bright field; 2 , 4: relief contrast); Crystallization experiments were carried out for $L$-glutamic acid $(1,2)$, mannitol $(3,4)$, and taurine (5). (b) Binary images after processing.

overlap and the challenges in applying the segmentation and watershed algorithms from a computational time and error point of view.

\subsection{Optimized Data Binning Approach}

The next challenge was the implementation of the image processing method described in Sect. 3.2, together with a data binning and statistical presentation strategy, in a practical fashion for large dataset tables. The data presentation approach ideally should have many features:

(a) to easily interpret size or shape distributions; (b) to efficiently distinguish the different species or populations; and (c) to present the evolutions in correlation and weighting of an ensemble of images rather than a single image (Fig. 8d).

It was not useful to draw the global average for each image as a data point on the curve (Fig. $8 \mathrm{~b}$ ), as such averages are strongly skewed by the number of microparticles and are not true averages due to the usually larger counts of small particles in each image. Certain images may contain 5-10 particles, while others up to 30-40 particles. However, by taking into consideration the particles from several images (i.e., every $X$ number of images such as $X=50$ or 100) and calculating the size-shape distribution, in terms of statistical analysis, there is a larger statistical representation and greater balance between data points in a way that each data point represents a particular size or shape range in those $X$ number of images. Such an approach (Fig. 8d) is also more guiding when compared to plotting every particle as a data point, for every image throughout the experiment (Fig. 8c). The latter does not provide a straightforward interpretation neither of the distribution, nor of the image reference.

Crystals of $L$-glutamic acid may adopt different forms and shapes $[29,30]$. Here, they first appeared as small needle forms, then adopted shapes that are multicrystalline (aggregates of multi-needles), with a basic urchin-like shape, which have a low solidity factor describing their shape (factor 0.5 to 0.7 ), compared to simple shapes of highly smooth and weakly convex/concave edges (maximum factor is 1 ).
From the application of this method of data binning, namely, calculating the size distribution, also applicable on shape factors, by plotting the frequency every 100 images of particles of $a-b \mu \mathrm{m}$ (e.g., $10-20 ; 50-100 ; 300-900$ ), it was possible to track the populations separately and their evolution on a time basis. In this case, e.g., particles of $50-100 \mu \mathrm{m}$ were forming until a later stage up to image $\# 300$, i.e., 3 rd data point, compared to smaller particles of less than $50 \mu \mathrm{m}$. Furthermore, particles of $100-300 \mu \mathrm{m}$ were increasing in counts, followed by particles of $300-900 \mu \mathrm{m}$. Towards the end of the experiment, there was a decrease in particle counts for several size populations due to the increase in temperature, causing dissolution.

A number of factors are found to be difficult to account for, such as: (a) microparticles which are dominated in the signal or overlapping with the larger particles, which may be causing the slight decrease in microparticle counts when the larger particles were increasing (e.g., after image \#200); (b) segmentation is not carried out here due to the limitation of computer image understanding, time to be imposed on our fast processing method, and the associated error when applied on particles of random shapes and rotations; (c) particles touching the image borders are not considered, which implies that those of larger sizes have a greater probability of touching the borders and have a larger probability of not being considered; and (d) finally, it is important to note that these values (Fig. 8) are count/number-weighted and not volume-weighted. This signifies that one sphere of $500 \mu \mathrm{m}$ in size (diameter), e.g., is equal in count to one sphere of $50 \mu \mathrm{m}$ (Fig. 9c.i), but the volumeweighted evaluation makes the former not 10 times larger in volume, but actually 1000 times. This again clarifies the importance of the larger particles of which, even though their counts are less, their volumes and statistical weight are significantly more dominant.

One additional advantage of the current method of presentation (Fig. 8d) is the possibility to combine all curves for all size intervals without a logarithmic or other transformation method, to fit into the same graph. Finally, the drop observed in Fig. $8 \mathrm{~d}$ is not complete compared to Fig. $8 \mathrm{~b}$, because the last data point in the former is calculated based on 100 images, 
a)
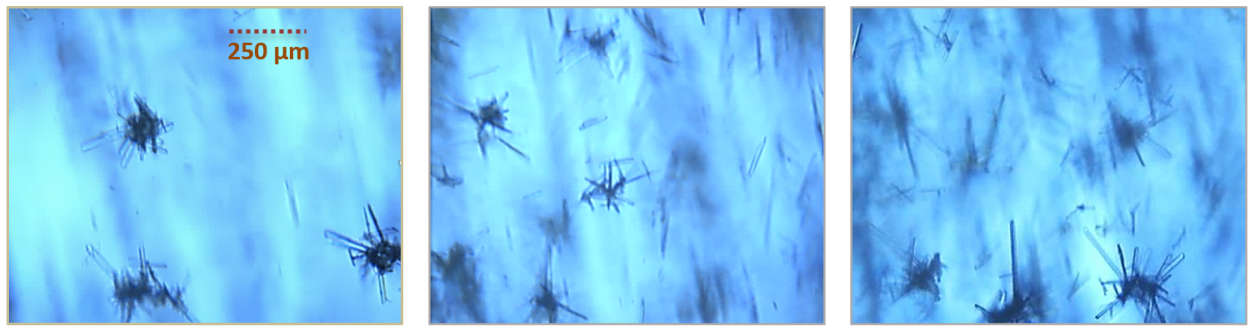

b) 120
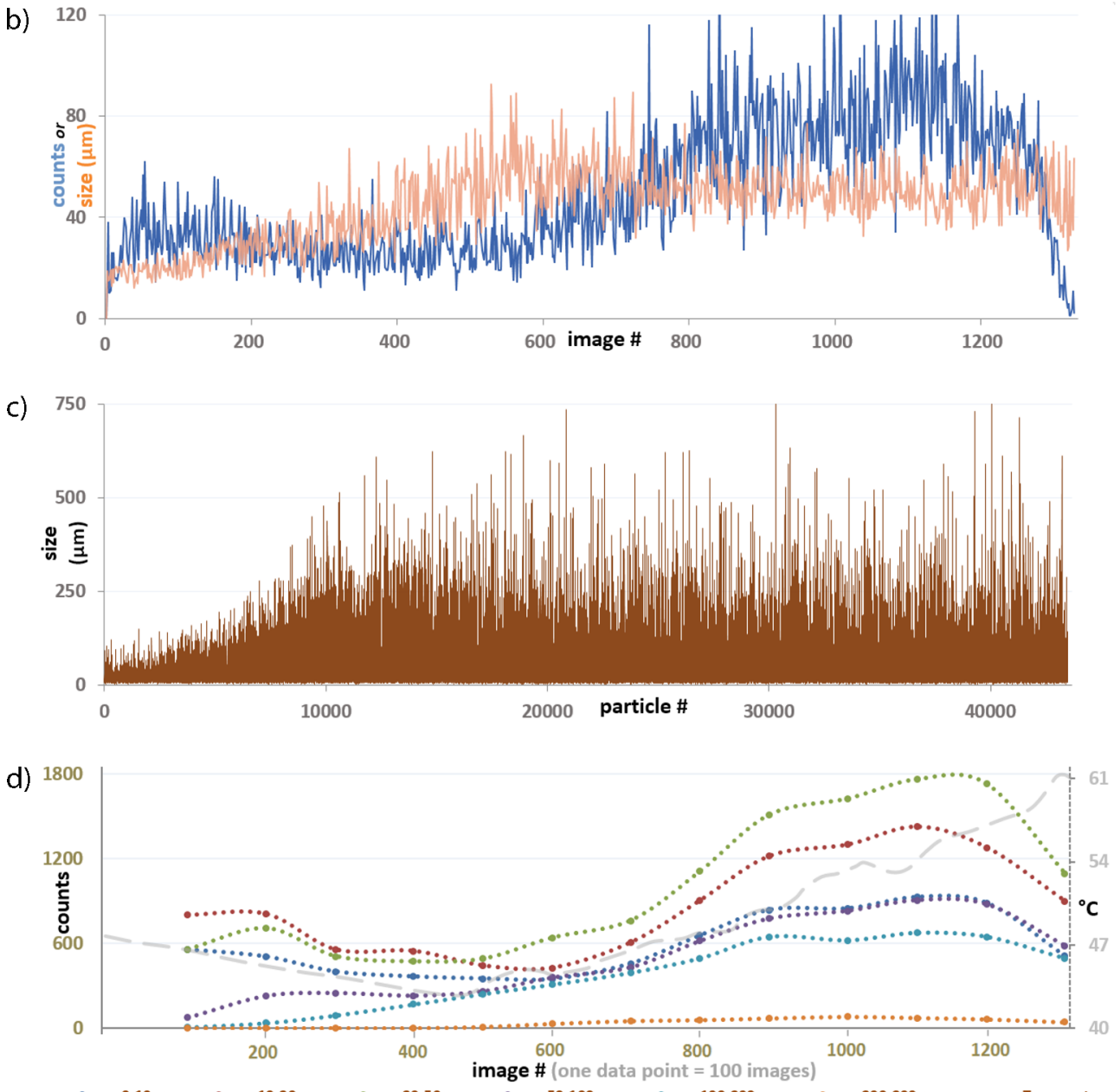

Figure 8. Data binning strategy for real-time monitoring of crystallization. (a) Selected raw images in bright field mode of fast L-glutamic acid crystallization (in situ); (b) average per image data presentation method, in which the particle counts as well as the average size (Feret's diameter) per image are shown for the entire image dataset. At the end there is dissolution due to the temperature increase (indicated in (d)). (c) Particle by particle data presentation method, in which the size of every particle is displayed (by order of images). (d) Data binning strategy, in which the frequency (count) of particles belonging to a customized size range is illustrated for every $X$ number of images (here $X=100$ images). In (d), data points that align vertically describe the particle distribution (e.g., 50-100 or $100-300 \mu \mathrm{m}$ ) for those particular 100 images, as number-weighted counts.

while in the latter it is based on overall averages per single images.

In this study, using microscopy which is the main method for shape analysis, it was possible to track the size-shape of particles while analyzing images via a single code along with a data binning approach. Furthermore, the direct observation of in situ images provides both a quantitative and qualitative evaluation of the binary analysis. Thus, the determined curves and trends in Fig. 8 for example and the preceding figures were reasonable. In previous studies $[19,20]$, the sensors (Fig. 2) and image analysis principle proved successful in determining sizes and shapes based on specific size-shape calculations of com- 
mercial particle size standards of known shapes, up to certain concentrations suitable for automated image analysis. Furthermore, in the current study, a comparison of the results by referring to other methods may not be rational, first, because video microscopy is usually considered as the only in situ method for shape determination, and second, because the principle of microscopy and image analysis is different from other methods' principles (e.g., chord length-based) which do not determine the shape but their measurements are highly dependent on the shape and orientation of particles (Fig. 9). Yet, each method has both advantages and disadvantages, depending on the concentration, resolution, and processing speed.

\section{Conclusion}

A straightforward approach was successful for processing inline images obtained using a PAT, and for the data classification and representation of large datasets containing multiple popu- lations of particles. The imaging algorithm strategy was applicable to the entire in situ image dataset and was rapid (10-30 s per image depending on the performance, image type, color/ hue requirement (i.e., Fig. 6)). The main advantage of the background correction method was further enhanced using the contrast enhancement function. Importantly, particle information was generally maintained, including micro and macro sizes and shapes. The contrast imaging is a step forward for the in-line characterization and to analyze certain texture information.

In addition to the direct image observation and interpretation, PAT imaging techniques can be employed to extract basic $1 \mathrm{D}$ and $2 \mathrm{D}$ information, as well as certain texture and shape details which can be used in future mechanistic models. Optimization of the imaging performance is necessary for high concentrations whereby challenges of segmentation and imaging performance limitation are encountered. Nevertheless, several of these challenges are encountered with other methods, such as particle overlap, signal saturation, and baseline limitations. Other PAT methodologies are found to encounter unwanted

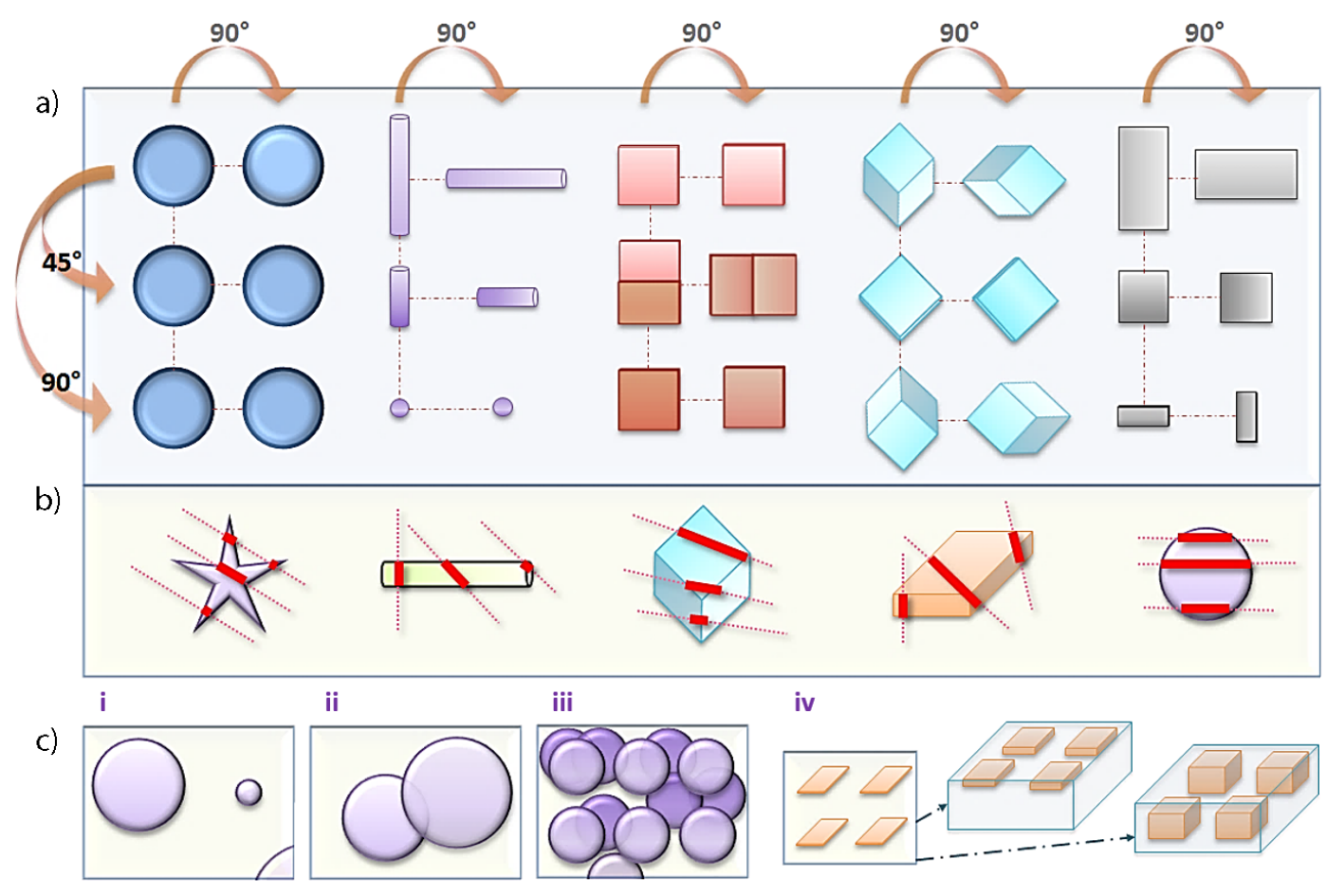

Figure 9. Particle size-shape factors and their impact on analysis results determined by 2D imaging compared to chord length measurement methods. (a) 2D imaging of particles observed according to different rotations. An extremely favorable shape is a sphere since it is observed identically from any angle, within an identical focal plane. For a needle (mannitol, Fig. 7), however, in the most extreme case, it may be seen as a circle/dot at $90^{\circ}$ rotation. (b) Chord length measurement of particles of different shapes. For a needle, there is low probability that the scan will be aligned along the full length. For particles of advanced shapes, chords detected are not helpful to presume the shape, and many shapes may result in a high number of false counts and size measurements. (c) Advanced features for future implementation in image-based size-shape analysis. (i) Volume-weighted data binning and not only count or number-weighted (e.g., large sphere is equal in count to a small sphere, but the volumetric contribution is a lot more significant). (ii) Considering whether an object is two (or more) overlapping particles, or a single polymorphic object. (iii) Image saturation is an obstacle to correctly determine the particle sizeshape due to overlap, turbidity, and change in image properties, which affects the estimation of concentration due to saturation of the field of view above a certain threshold. (iv) Impact of object's shape on the volumeweighted contribution and observed concentration. For instance, similar objects may appear the same in 2D projections, but one population is many-fold larger in 3D volume. 
effects including chord concatenation, chord splitting, refractive index issues, etc.

A realistic representation method was achieved (Fig. 8) using groups of images and by calculating the PSD within, i.e, frequencies within intervals of specific size or shape ranges. The approach is important for large datasets. Further developments are possible through a volume rather than number-weighted representation, by taking into consideration shape and size to represent the real physical contribution and evolution of large populations in comparison to microparticles. In fact, the shape descriptors (Figs. 4 and 5) and surface areas may also facilitate the volumetric calculation. Such volume estimations may be further improved using models for the probabilities of rotation of particles respective to the camera [31], e.g., visualization of $3 \mathrm{D}$ particles in $2 \mathrm{D}$ depends on their rotations and projections towards the camera (Fig. 9). The latter is a common challenge in several physical characterization methods, especially those measuring in $1 \mathrm{D}$.

The strategy developed offers the potential for increased control and automation of crystallization processes. It could provide a feedback strategy and decision-making tool in addition to the direct, classic monitoring function. The approach may be also improved for more general applications using sophisticated machine-vision processing steps and neural networks [32].

\section{Supporting Information}

Supporting Information for this article can be found under DOI: https://doi.org/10.1002/ceat.201900311.

\section{Acknowledgment}

Research leading to many results has received funding from the European Community's Seventh Framework Program (FP7-SME-2013) under the CRYSTAL-VIS project, grant agreement number 605814, and from Science Foundation Ireland (SFI) through a Technology Innovation Development Award (TIDA). The authors would like to thank the project partners, Innopharma Technology (Ireland), the PAT group (TU Dublin, Ireland), VTT (Finland), APC Ltd (Ireland) and Labiana Pharmaceuticals, S.L.U. (Spain).

The authors have declared no conflict of interest.

\section{Abbreviations}

PAT process analytical technology

PBM population balance model

QbD quality by design

\section{References}

[1] F. F. Gouveia, J. P. Rahbek, A. R. Mortensen, M. T. Pedersen, P. M. Felizardo, R. Bro, M. J. Mealy, Anal. Bioanal. Chem.
2017, 409 (3), 821-832. DOI: https://doi.org/10.1007/ s00216-016-9834-Z

[2] Y. Qiao, R. Qiao, Y. He, C. Shi, Y. Liu, H. Hao, J. Su, J. Zhong, Cryst. Growth Des. 2017, 17, 6138-6148. DOI: https:// doi.org/10.1021/acs.cgd.7b00759

[3] M. R. Abu Bakar, Z. K. Nagy, C. D. Rielly, Cryst. Growth Des. 2010, 10 (9), 3892-3900. DOI: https://doi.org/10.1021/ cg1002379

[4] R. Irizarry, A. Chen, R. Crawford, L. Codan, J. Schoell, Chem. Eng. Sci. 2017, 164, 202-218. DOI: https://doi.org/ 10.1016/j.ces.2017.01.042

[5] N. Kail, H. Briesen, W. Marquardt, Part. Part. Syst. Char. 2007, 24 (3), 184-192. DOI: https://doi.org/10.1002/ ppsc. 200601036

[6] A. Tadayyon, S. Rohani, Part. Part. Syst. Char. 1998, 15 (3), 127-135. DOI: https://doi.org/10.1002/(SICI) 1521-4117(199817)15:3<127::AID-PPSC127>3.0.CO;2-B

[7] Y. Huo, T. Liu, X. Z. Wang, C. Y. Ma, X. Ni, Ind. Eng. Chem. Res. 2017, 56 (39), 11257-11269. DOI: https://doi.org/ 10.1021/acs.iecr.7b02439

[8] A. Amon, P. Born, K. E. Daniels, J. A. Dijksman, K. Huang, D. Parker, M. Schröter, R. Stannarius, A. Wierschem, Rev. Sci. Instrum. 2017, 88 (5), 051701. DOI: https://doi.org/ $10.1063 / 1.4983052$

[9] A. Mehle, B. Likar, D. Tomaževič, IPSJ Trans. Comput. Vision Appl. 2017, 9 (1), 7. DOI: https://doi.org/10.1186/ s41074-017-0019-2

[10] J. Aarthi, P. Dhanasekaran, T. S. Senthil, N. M. Ganesan, Cryst. Res. Technol. 2018, 53 (6), 1700190. DOI: https:// doi.org/10.1002/crat.201700190

[11] R. Kacker, M. Radoiu, H. J. M. Kramer, Cryst. Growth Des. 2017, 17 (7), 3766-3774. DOI: https://doi.org/10.1021/ acs.cgd.7b00368

[12] T. Kodera, M. Kobari, I. Hirasawa, Chem. Eng. Technol. 2019, 42 (7), 1458-1465. DOI: https://doi.org/10.1002/ ceat. 201800693

[13] W. Yu, L. Liao, R. Bharadwaj, B. C. Hancock, Powder Technol. 2017, 313, 1-8. DOI: https://doi.org/10.1016/ j.powtec.2017.02.043

[14] S. M. Miller, J. B. Rawlings, AIChE J. 1994, 40 (8), 1312 1327. DOI: https://doi.org/10.1002/aic.690400805

[15] B. Szilágyi, B. G. Lakatos, Comput. Chem. Eng. 2017, 98 , 180-196. DOI: https://doi.org/10.1016/ j.compchemeng.2016.11.028

[16] N. A. F. A. Samad, G. Sin, K. V. Gernaey, R. Gani, Eur. J.6nbsp;Pharm. Biopharm. 2013, 85 (3, Part B), 911-929. DOI: https://doi.org/10.1016/j.ejpb.2013.05.016

[17] M. Jiang, X. Zhu, M. C. Molaro, M. L. Rasche, H. Zhang, K. Chadwick, D. M. Raimondo, K.-K. K. Kim, L. Zhou, Z. Zhu, M. H. Wong, D. O'Grady, D. Hebrault, J. Tedesco, R. D. Braatz, Ind. Eng. Chem. Res. 2014, 53 (13), 5325-5336. DOI: https://doi.org/10.1021/ie400859d

[18] S. Ho-Van, B. Bouillot, D. Garcia, J. Douzet, A. Cameirao, S. Maghsoodloo-Babakhani, J.-M. Herri, Chem. Eng. Technol. 2019, 42 (7), 1481-1491. DOI: https://doi.org/10.1002/ ceat. 201800746

[19] T. El Arnaout, P. J. Cullen, C. Sullivan, Chem. Eng. Sci. 2016, 149, 42-50. DOI: https://doi.org/10.1016/j.ces.2016.04.025 
[20] T. El Arnaout, L. Kurki, T. Vaarala, K. Ojala, P. J. Cullen, C. Sullivan, Chem. Eng. J. 2016, 300, 64-74. DOI: https:// doi.org/10.1016/j.cej.2016.04.126

[21] T. El Arnaout, P. J. Cullen, Sci. Rep. 2017, 7 (1), 6384. DOI: https://doi.org/10.1038/s41598-017-06830-8

[22] R. Danz, A. Vogelgsang, R. Käthner, C. Zeiss, G. Plant, PlasDIC - a useful modification of the differential interference contrast according to Smith/Nomarski in transmitted light arrangement, Photonik 2004. https://pdfs.semanticscholar. org/8475/7ffa6a6a2e795063a2760aa0d11a7863f90e.pdf

[23] S. R. Sternberg, Computer 1983, 16 (1), 22-34. DOI: https:// doi.org/10.1109/MC.1983.1654163

[24] C. A. Schneider, W. S. Rasband, K. W. Eliceiri, Nat. Methods 2012, 9 (7), 671-675.

[25] N. N. Misra, R. Phalak, A. Martynenko, J. Food Eng. DOI: https://doi.org/10.1016/j.jfoodeng.2018.06.007

[26] H. Zhu, F. H. Y. Chan, F. K. Lam, Computer Vision and Image Understanding 1999, 73 (2), 281-290. DOI: https:// doi.org/10.1006/cviu.1998.0723
[27] C. Lim, H.-S. Kim, K.-J. Kim, Chem. Eng. Technol. 2017, 40 (7), 1309-1317. DOI: https://doi.org/10.1002/ ceat. 201700128

[28] O. S. Agimelen, P. Hamilton, I. Haley, A. Nordon, M. Vasile, J. Sefcik, A. J. Mulholland, Chem. Eng. Sci. 2015, 123, 629640. DOI: https://doi.org/10.1016/j.ces.2014.11.014

[29] Y. Tahri, E. Gagnière, E. Chabanon, T. Bounahmidi, D. Mangin, J. Cryst. Growth 2016, 435, 98-104. DOI: https://doi.org/ 10.1016/j.jcrysgro.2015.11.019

[30] Z. Gao, Y. Wu, Y. Bao, J. Gong, J. Wang, S. Rohani, Cryst. Growth Des. 2018, 18 (8), 4275-4281. DOI: https://doi.org/ 10.1021/acs.cgd.8b00883

[31] J. Cardona, C. Ferreira, J. McGinty, A. Hamilton, O. S. Agimelen, A. Cleary, R. Atkinson, C. Michie, S. Marshall, Y.-C. Chen, J. Sefcik, I. Andonovic, C. Tachtatzis, Chem. Eng. Sci. 2018, 191, 208-231. DOI: https://doi.org/10.1016/ j.ces.2018.06.067

[32] S. Heisel, M. Rolfes, K. Wohlgemuth, Chem. Eng. Technol. 2018, 41 (6), 1218-1225. DOI: https://doi.org/10.1002/ ceat. 201700651 